\title{
Beginner's guide to comparative bacterial genome analysis using next-generation sequence data
}

\author{
David J Edwards ${ }^{1,2}$ and Kathryn E Holt ${ }^{*}$
}

\begin{abstract}
High throughput sequencing is now fast and cheap enough to be considered part of the toolbox for investigating bacteria, and there are thousands of bacterial genome sequences available for comparison in the public domain. Bacterial genome analysis is increasingly being performed by diverse groups in research, clinical and public health labs alike, who are interested in a wide array of topics related to bacterial genetics and evolution. Examples include outbreak analysis and the study of pathogenicity and antimicrobial resistance. In this beginner's guide, we aim to provide an entry point for individuals with a biology background who want to perform their own bioinformatics analysis of bacterial genome data, to enable them to answer their own research questions. We assume readers will be familiar with genetics and the basic nature of sequence data, but do not assume any computer programming skills. The main topics covered are assembly, ordering of contigs, annotation, genome comparison and extracting common typing information. Each section includes worked examples using publicly available E. coli data and free software tools, all which can be performed on a desktop computer.
\end{abstract}

Keywords: Bacterial, Microbial, Comparative, Genomics, Next generation sequencing, Analysis, Methods

\section{Review}

\section{Introduction and aims}

High throughput sequencing is now fast and cheap enough to be considered part of the toolbox for investigating bacteria $[1,2]$. This work is performed by diverse groups of individuals including researchers, public health practitioners and clinicians, interested in a wide array of topics related to bacterial genetics and evolution. Examples include the study of clinical isolates as well as laboratory strains and mutants [3]; outbreak investigation $[4,5]$; and the evolution and spread of drug resistance [6]. Bacterial genome sequences can now be generated in-house in many labs, in a matter of hours or days using benchtop sequencers such as the Illumina MiSeq, Ion Torrent PGM or Roche 454 FLX Junior [1,2]. Much of this data is available in the public domain, allowing for extensive comparative analysis; e.g. in February 2013 the GenBank database included $>6,500$ bacterial genome

\footnotetext{
*Correspondence: kholt@unimelb.edu.au

'Department of Biochemistry and Molecular Biology, Bio21 Institute, University of Melbourne, Victoria 3010, Australia

Full list of author information is available at the end of the article
}

assemblies, two thirds of which were in 'draft' form (i.e. presented as a set of sequence fragments rather than a single sequence representing the whole genome, see [7] for a detailed discussion).

In this beginner's guide, we aim to provide an entry point for individuals wanting to make use of wholegenome sequence data for the de novo assembly of genomes to answer questions in the context of their broader research goals. The guide is not aimed at those wishing to perform automated processing of hundreds of genomes at a time; some discussion of the use of sequencing in routine microbiological diagnostic laboratories is available in the literature [8]. We assume readers will be familiar with genetics and the basic nature of sequence data, but do not assume any computer programming skills and all the examples we use can be performed on a desktop computer (Mac, Windows or Linux). The guide is not intended to be exhaustive, but to introduce a set of simple but flexible and free tools that can be used to investigate a variety of common questions including (i) how does this genome compare to that one?, and (ii) does this genome have plasmids,

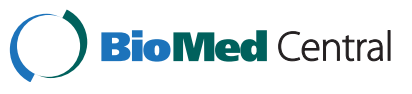


phage or resistance genes? Each section includes guidance on where to find more detailed technical information, alternative software packages and where to look for more sophisticated approaches.

\section{Examples and tutorial}

Throughout the guide, we will use Escherichia coli O104:H4 as a worked example. E. coli O104:H4 was responsible for a lethal foodborne outbreak of haemolytic uraemic syndrome (HUS) in Germany during 2011 [9-11]. Sequence reads and assemblies from a number of outbreak strains, generated using different high throughput sequencing platforms (including Illumina, Ion Torrent and 454) are now available for download from the European Nucleotide Archive [11-17].

The outbreak strain belongs to an enteroaggregative $E$. coli (EAEC) lineage that has acquired a bacteriophage encoding Shiga-toxin (commonly associated with enterohaemorrhagic E. coli (EHEC)), and multiple antibiotic resistance genes [12]. For the worked examples, we will use a set of paired-end Illumina reads from O104:H4 strain TY-2482 (ENA accession SRR292770), but also include alternatives for the other available short-read data types. For those so interested, longer Pacific Bioscience reads are also available, but are not included in this tutorial.

The workflow has been divided into five logical sections: assembly, ordering of contigs, annotation, genome comparison and typing. Examples using E. coli O104:H4 data are presented in the text and figures, and detailed instructions on how to replicate the example are provided in the corresponding tutorial (Additional file 1). The tutorial includes links to the software programs required for each stage, the specific steps needed to use the program(s), and the expected inputs and outputs (instructions for software installation are provided by the developers of each program).

Whilst quality control of raw sequence data can be important in obtaining the best assembly for comparison, the number and complexity of possible steps is too numerous, and the variations between platforms too substantial, to cover in this guide. However, we recommend readers check the quality of raw sequence reads using the tools accompanying their benchtop sequencing machines, or use FastQC to assess the quality of raw read sets (see Tutorial, Additional file 1).

\section{Genome assembly}

De novo assembly is the process of merging overlapping sequence reads into contiguous sequences (contigs) without the use of any reference genome as a guide (Figure 1). The most efficient assemblers for short-read sequences are typically those that employ de Bruijn graphs to produce an assembly [18]. An eloquent explanation of how de Bruijn graphs work in sequence assembly can be found in Compeau et al. [18]. One of the first and most widely used de Bruijn graph assemblers is the open-source

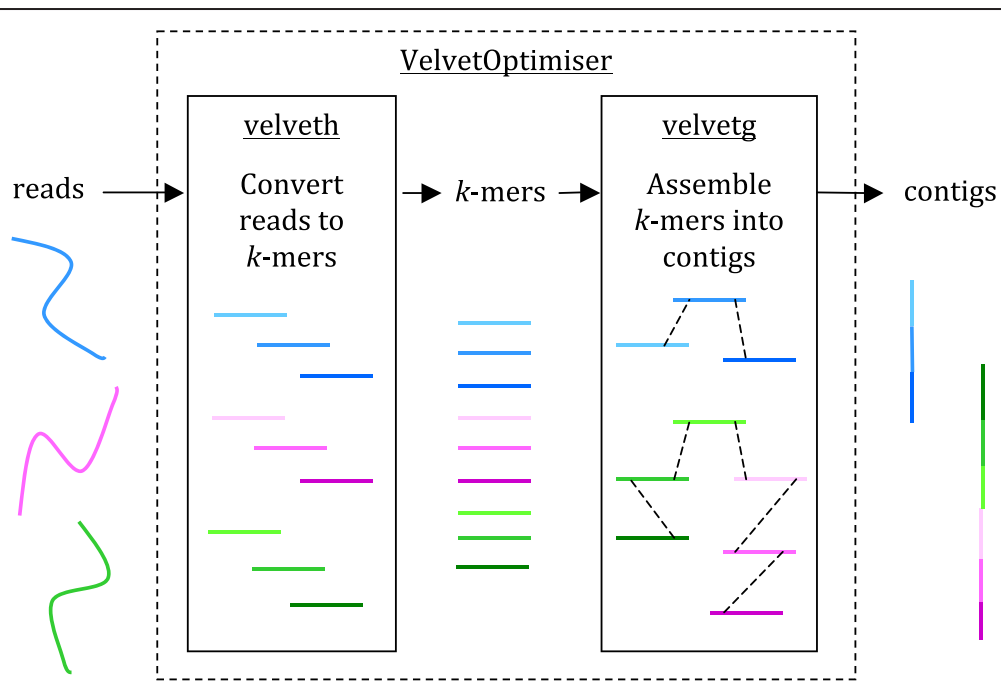

Figure 1 Genome assembly with Velvet. Reads are assembled into contigs using Velvet and VelvetOptimiser in two steps, (1) velveth converts reads to $k$-mers using a hash table, and (2) velvetg assembles overlapping $k$-mers into contigs via a de Bruijn graph. VelvetOptimiser can be used to automate the optimisation of parameters for velveth and velvetg and generate an optimal assembly. To generate an assembly of $E$. coli 0104 : H4 using the command-line tool Velvet: • Download Velvet [23] (we used version 1.2.08 on Mac OS X, compiled with a maximum $k$-mer length of 101 bp) • Download the paired-end Illumina reads for E. coli O104:H4 strain TY-2482 (ENA accession SRR292770 [17]) • Convert the reads to k-mers using this command: velveth out_data_35 35 -fastq.gz -shortPaired -separate SRR292770_1.fastq.gz SRR292770_2.fastq.gz • Then, assemble overlapping $k$-mers into contigs using this command: velvetg out_data_35 -clean yes -exp_cov 21 -cov_cutoff 2.81 -min_contig_lgth 200 This will produce a set of contigs in multifasta format for further analysis. See Additional file 1: Tutorial for further details, including help with downloading reads and using VelvetOptimiser. 
program Velvet [19]. With further development to improve the resolution of repeats and scaffolding using paired-end and longer reads [20], Velvet remains one of the most-used (and cited) assemblers for bacterial genomes, being best suited to Illumina sequence reads (Velvet is included as the default assembler in the Illumina MiSeq analysis suite).

Ion Torrent reads are better assembled using the open source program MIRA [21], which uses a modified SmithWaterman algorithm for local alignment rather than a de Bruijn graph method. MIRA is available as a plugin for the Ion Torrent analysis suite. For 454 data, Roche provides a proprietary (de Bruijn graph-based) assembler [22].

When using a de Bruijn graph assembler, a number of variables need to be considered in order to produce optimal contigs [23]. This can be automated quite effectively using VelvetOptimiser [24]. The key issue is selecting an appropriate $k$-mer length for building the de Bruijn graph. Different sequencing platforms produce fragments of differing length and quality [1], meaning very different ranges of $k$-mers will be better suited to different types of read sets. A balance must be found between the sensitivity offered by a smaller $k$-mer against the specificity of a larger one [18]. Other variables to consider when running Velvet include the expected coverage across the genome, the length of the insert sizes in paired-end read libraries, and the minimum coverage (read depth) cut-off value, all of which can be automated using VelvetOptimiser [23]. If the coverage obtained is higher than $20 \times$ reads deep on average, the chances of errors being incorporated into the contigs increases, as de Bruijn graph assemblers cannot distinguish between an error and a real variant if there is lots of evidence for the error, as found with higher coverage levels. In this case, a subset of the reads can be sampled and used for the assembly [23].

Instructions on how to assemble Illumina reads from E. coli $\mathrm{O} 104: \mathrm{H} 4$ strain TY-2482 using Velvet are given in Figure 1 and Additional file 1: Tutorial. The assembler takes the sequence reads as input (in fastq format) and outputs the assembled contigs (in multifasta format). Note that the contig set, referred to as the draft assembly, will include sequences derived from all the DNA present in the sequenced sample, including chromosome (s) and any bacteriophage or plasmids.

\section{Ordering and viewing assembled contigs}

Once a set of contigs have been assembled from the sequencing reads, the next step is to order those contigs against a suitable reference genome. This may seems counter-intuitive at first as we have applied de novo assembly to obtain these contigs, but ordering the contigs aids the discovery and comparison process. The best reference to use is usually the most closely related bacterium with a 'finished' genome, but as in the case of $E$. coli
O104:H4, finding the best reference may itself involve trial and error [12].

Ordering of contigs can be achieved using commandline tools such as MUMmer [25], which can be simplified using a wrapper program like $A B A C A S$ [26]. However we suggest the easiest way for beginners is to use the contig ordering tool in the Java-based graphical-interface program Mauve [27,28]. This ordering algorithm uses an iterative mapping approach to find the best fit for each contig against the reference genome. Mauve takes as input the reference genome in fasta format along with the assembly in multifasta format, and outputs another multifasta file containing the ordered contigs. Detailed instructions for ordering the E. coli O104:H4 contigs against a reference are given in Additional file 1: Tutorial.

Due to evolutionary differences between the reference and novel genome, the presence of (often mobile) repeat elements such as prophages, and the very nature of short-read assemblers, there will almost certainly be assembly errors present within the contigs. Indeed, all assemblers used in Assemblathon 1 [29] and the Genome Assembly Gold-standard Evaluations (GAGE) [30] community "bake-offs" produced assemblies with errors. The error rate of an assembly can be assessed if a closely related reference genome is available. A good option for assessing the error rate is MauveAssemblyMetrics [31] (see Additional file 1: Tutorial for an example with E. coli $\mathrm{O} 104: \mathrm{H} 4)$, an optional addition to Mauve that generates a report on assembly quality.

Another way to explore the ordered assembly is by means of visualization. Mauve provides one way to visualize the assembly by alignment to other sequences (see Additional file 1: Tutorial for instructions). Another option is to use Artemis and the companion Artemis Comparison Tool (ACT), a pair of open-source Javabased applications [32]. An example using E. coli O104: $\mathrm{H} 4$ is shown in Figure 2 and in Additional file 1: Tutorial. To view comparisons in $A C T$, you need to first generate a comparison file that identifies regions of homology between your assembly and a reference genome. You can then load this into ACT along with your assembly and reference sequence(s). The comparison file can be generated using the WebACT or DoubleACT websites, or using $B L A S T+$ on your own computer (see Additional file 1: Tutorial for details of these programs). Note that before you can generate the comparison file, the assembly needs to be converted into a single fasta sequence. This can be done in Artemis (Figure 2), or using a command-line tool such as the 'union' command in the EMBOSS package [33] (see Additional file 1: Tutorial for details).

\section{Genome annotation}

Once the ordered set of contigs has been obtained, the next step is to annotate the draft genome. Annotation is 


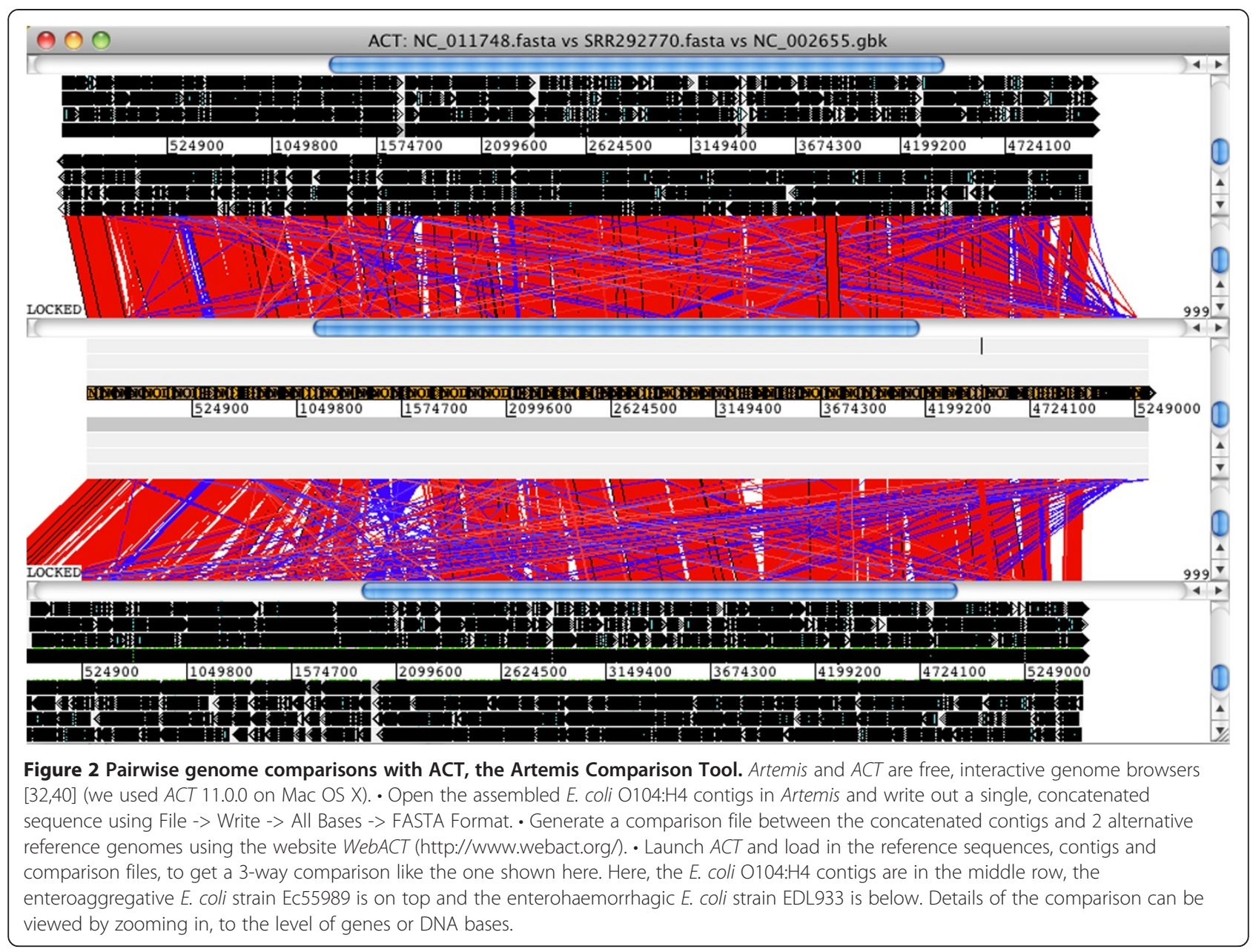

the process of 'gene' finding, and can also include the identification of ribosomal and transfer RNAs encoded in the genome. Bacterial genome annotation is most easily achieved by uploading a genome assembly to an automated web-based tool such as RAST [34,35]. There are also many command-line annotation tools available. These include methods based on de novo discovery of genes, such as Prokka [36] and DIYA [37], or programs that transfer annotation directly from closely related genomes, such as RATT [38] and BG-7 [39].

Since the quality of the final annotation is largely determined by the quality of the gene database used, we prefer the easy-to-use online de novo annotation tool RAST for bacterial genome annotation [35]. RAST takes as input the ordered contigs in multifasta format, identifies open reading frames that are likely to be genes, and uses a series of subsystem techniques (the 'ST' in RAST) to compare these with a sophisticated database of genes and RNA sequences, producing a high-quality annotation of the assembly. The genes identified can be viewed, and compared to other genomes, using the RAST online tool. The annotation can also be downloaded in a variety of formats, including in GenBank format. See Additional file 1: Tutorial for detailed instructions on how to annotate the E. coli O104:H4 genome using RAST.

\section{Comparative genome analysis}

For most sequencing experiments, comparison to other genomes or sequences is a critical step. Sometimes general questions are asked, along the lines of "which genes do these genomes share and which are unique to particular genomes?". In many cases, users are also interested in looking for specific genes that are known to have important functions, such as virulence genes or drug resistance determinants.

For most users, it is important to be able to visualize these comparisons, both to aid understanding and interpretation of the data, and to generate figures for communicating results. We therefore recommend three software tools that combine data analysis and visualization - BRIG, Mauve and ACT (the latter two have already been introduced above). For more experienced users, comparative questions can also be answered using command-line search tools, such as MUMmer or BLAST. 
$A C T[32,40]$ is a Java-based tool for visualizing pairwise comparisons of sequences, including whole genomes. As outlined above, BLAST is used to compare the sequences (this can be done locally, or through web services); the two genomes and the BLAST result are then loaded into $A C T$ for visualization of the comparison (see Additional file 1: Tutorial). Multiple pairwise comparisons can be visualized simultaneously; an example using E. coli O104:H4 is given in Figure 2 and Additional file 1: Tutorial. Regions of sequence homology are linked by blocks, which are coloured red (same orientation) or blue (reverse orientation), with saturation indicating the degree of homology (dark=high homology, to light=low homology). Advantages of using $A C T$ include (i) the flexibility to zoom right out to see whole-genome comparisons, (ii) ability to zoom right down to DNA and/or protein sequences to examine fine-scale comparisons, and (iii) it is possible to add or edit annotations for the genomes being compared.

Mauve is a Java-based tool for multiple alignment of whole genomes, with a built-in viewer and the option to export comparative genomic information in various forms $[27,41]$. Its alignment functions can also be used to order and orient contigs against an existing assembly, as outlined above. Mauve takes as input a set of genome assemblies, and generates a multiple whole-genome alignment. It identifies blocks of sequence homology, and assigns each block a unique colour. Each genome can then be visualized as a sequence of these coloured sequence blocks, facilitating visualization of the genome comparisons. An example is given in Figure 3. This makes it easy to identify regions that are conserved among the whole set of input genomes, and regions that are unique to subsets of genomes (islands). The tutorial (Additional file 1) includes a detailed example of how to use Mauve to identify unique regions in the E. coli O104:H4 outbreak assembly compared to EHEC and EAEC chromosomal sequences. Because Mauve generates an alignment of the genome sequences, it can also be used to identify single nucleotide polymorphisms (SNPs, or point mutations) suitable for downstream phylogenetic or evolutionary analyses (see the Mauve user guide for details).

BRIG, or the BLAST Ring Image Generator, is a Javabased tool for visualizing the comparison of a reference sequence to a set of query sequences $[42,43])$. Results are plotted as a series of rings, each representing a query sequence, which are coloured to indicate the presence of hits to the reference sequence (see Figure 4). BRIG is flexible and can be used to answer a broad range of comparative questions, depending on the selection of the reference and comparison sequences. However it is important to keep in mind that this particular approach is reference-based, meaning it can show you which regions of the reference sequence are present or absent in query sequences, but it cannot reveal regions of the query sequences that are missing from the reference sequence. Therefore the selection of the reference is critical to understanding the results. An example is given in Figure 4, in which an EHEC genome is used as the reference sequence and the E. coli O104:H4 outbreak genome assembly, along with other pathogenic E. coli genomes, are used as queries. This makes it easy to see that the outbreak strain differs significantly from enterohaemorrhagic E. coli (EHEC) in terms of gene content, but shares with

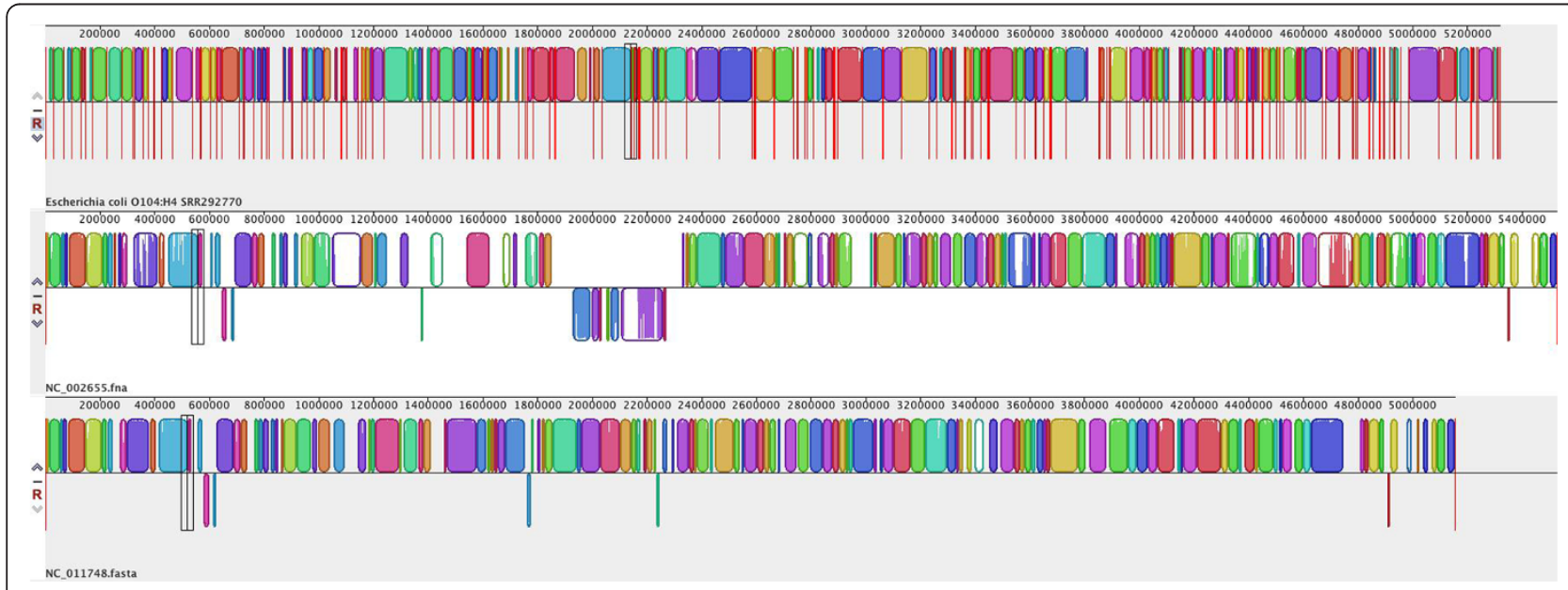

Figure 3 Mauve for multiple genome alignment. Mauve is a free alignment tool with an interactive browser for visualising results [27,41] (we used Mauve 2.3.1 on Mac OS X). • Launch Mauve and select File -> Align with progressiveMauve • Click 'Add Sequence...' to add your genome assembly (e.g. annotated E. coli O104:H4 contigs) and other reference genomes for comparison. • Specify a file for output, then click 'Align. ..' • When the alignment is finished, a visualization of the genome blocks and their homology will be displayed, as shown here. E. coli O104:H4 is on the top, red lines indicate contig boundaries within the assembly. Sequences outside coloured blocks do not have homologs in the other genomes. 


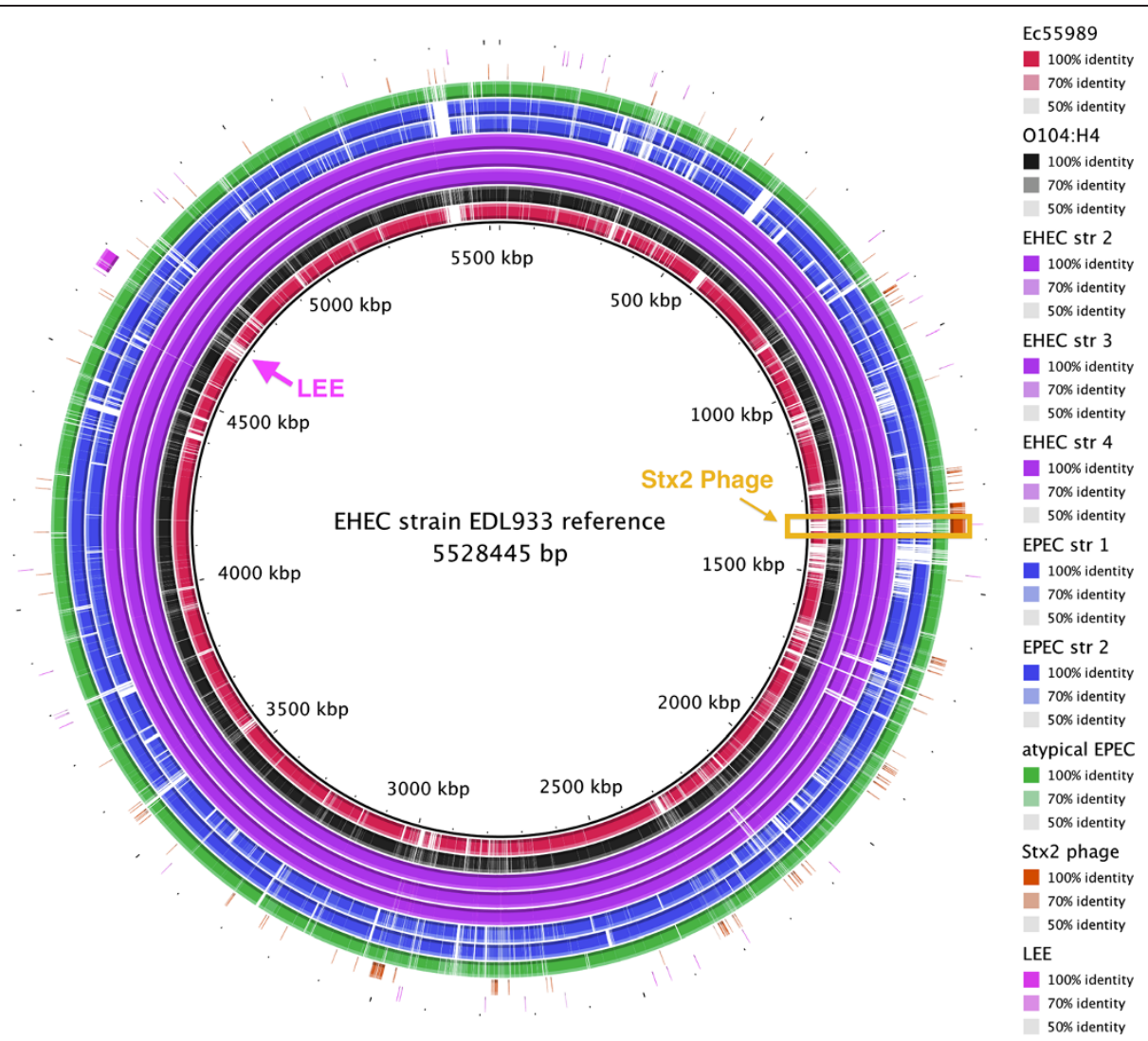

Figure 4 BRIG for multiple genome comparison. BRIG is a free tool $[42,43]$ that requires a local installation of BLAST (we used BRIG 0.95 on Mac OS X). The output is a static image. - Launch BRIG and set the reference sequence (EHEC EDL933 chromosome) and the location of other $E$. coli sequences for comparison. If you include reference sequences for the Stx2 phage and LEE pathogenicity island, it will be easy to see where these sequences are located. - Click 'Next' and specify the sequence data and colour for each ring to be displayed in comparison to the reference. - Click 'Next' and specify a title for the centre of the image and an output file, then click 'Submit' to run BRIG. BRIG will create an output file containing a circular image like the one shown here. It is easy to see that the Stx2 phage is present in the EHEC chromosomes (purple) and the outbreak genome (black), but not the EAEC or EPEC chromosomes.

it the Stx2 phage sequence which is missing from enteroaggregative E. coli (EAEC) and enteropathogenic E. coli (EPEC) (highlighted in Figure 4). The tutorial includes a second example, using the E. coli $\mathrm{O} 104: \mathrm{H} 4$ outbreak genome as the reference for comparison.

\section{Typing and public health applications: identifying} resistance genes, sequence types, phage, plasmids and other specific sequences

Whole genome sequencing is increasingly being used in place of PCR-based sequencing or typing methods. Here we outline some specialist tools for these purposes. The tutorial contains instructions for using these tools to examine the E. coli O104:H4 outbreak genome.

The detection of antimicrobial resistance genes is a key question for many researchers, especially in public health and diagnostic labs. The ResFinder tool [44], freely available online [45], allows users to upload sequence data to search against its curated database of acquired antimicrobial resistance genes. Sequence search is performed by BLAST, and the output is displayed in a table format that indicates which resistance genes were found, where they were found (contig name and coordinates), and the expected effect on phenotype. The fastest way to use ResFinder is to upload a genome assembly, however it is also possible to upload raw sequence reads in fastq format, which will be assembled prior to searching for resistance genes.

Multi-locus sequence typing (MLST) is a widely used, sequence-based method for typing of bacterial species and plasmids [46]. In February 2013, public MLST schemes were available for over 100 bacterial species and five plasmid incompatibility types [47]. The Center for Genomic Epidemiology hosts a publicly available web-based tool [48] that allows users to upload sequence data and extract sequence types for most of the publicly available MLST schemes. Like ResFinder, the tool uses BLAST searches of assemblies to identify sequence types, and can accept either genome assemblies or read sets, which are assembled on the fly prior to searching. Sequence types can also be 
extracted directly from reads, which can be more sensitive than assembly; see e.g. SRST, a command-line tool based on read mapping $[49,50]$.

For many bacteria, phage are the most dynamic part of the genome and are therefore of key interest to many researchers. Several free online tools exist for the identification of prophage sequences within bacterial genomes. A particularly feature-rich tool is PHAST (PHAge Search Tool) [51]. Genome assemblies can be uploaded in fasta or GenBank format; outputs include summary tables (indicating the location and identity of phage sequences within the assembly) and interactive tools for visualization of both the individual phage annotations and their locations on a circular map of the genome.

In most bacterial genome sequencing experiments, whole genomic DNA is extracted from the isolate and thus the sequence data includes both chromosomal and plasmid DNA. Many researchers are interested in exploring which plasmids are present in their bacterial genomes, particularly in the context of plasmid-borne resistance genes or virulence genes. One approach to rapidly detecting the presence and sequence type of a particular plasmid incompatibility group is to run a plasmid MLST analysis, e.g. using SRST [49]. However this will only work for the small number of plasmids with MLST schemes, and does not tell you which genes are encoded in the plasmid.

The ability to determine which sequences belong to plasmids and which belong to chromosomes varies with each sequencing experiment. This generally hinges on whether it is possible to assemble whole plasmids into a single sequence, which depends on many factors including read length, the availability of paired-end or matepair data, and the presence of repetitive DNA within the plasmid sequence. In most cases it is not possible to confidently assign every single contig to its correct replicon (i.e. chromosome or specific plasmid), without performing additional laboratory experiments. However, it is possible to get a very good idea of what plasmids are present in a genome assembly using comparative analyses. A good place to start is to identify all the contigs that are not definitely chromosomal (by comparing to other sequenced chromosomes using $A C T$ or Mauve, see above) and BLAST these against GenBank or a plasmid-specific database. One such database is available on the PATRIC website [52]. On the PATRIC BLAST page, select 'blastn' from the Program dropdown list and select 'Plasmid sequences' from the Database dropdown list. At the bottom of the page you can choose to view your results graphically (great if you are just searching a few contigs) or as a table (better if you have lots of contigs to investigate). The most similar plasmid sequences should make good candidates for more detailed comparison and visualization using Mauve, $A C T$ or BRIG as outlined above.
Another useful approach is to perform a blastn (nucleotide BLAST) search of the whole database at NCBI to see which known sequences your non-chromosomal contigs match (go to [53] and click 'nucleotide blast', then upload your contigs and make sure you are searching the 'nr' database). If you find you have a large contig with lots of matches to plasmid sequences, it's likely your contig is also part of a plasmid. One advantage of using NCBI's BLAST search page is that results can be viewed in the form of a phylogenetic tree (click 'Distance tree of results' at the top of the results page). This can help to quickly identify the plasmid sequences closest to yours, which can then be used for comparative analysis. If you find a contig that has close matches to part of a known plasmid, it may be of interest to know if the rest of the reference plasmid sequence is also present in the novel genome. You could get a quick idea of this using BRIG - use the known plasmid sequence as the reference and your set of assembled contigs as the query, then look to see how much of the known plasmid is covered by contigs. If more of the plasmid is covered, an ACT comparison could be performed using the reference plasmid and the annotated contig set, in order to identify which other contigs are likely to 'belong' to the same plasmid replicon and inspect what other genes are carried by the new plasmid.

\section{Other analyses}

There are many other methods for performing comparative bacterial genomic analysis, which are not discussed here. In particular, we have not discussed phylogenetic analysis, or how to perform detailed gene content comparisons between sets of genomes.

Arguably, phylogenetic analysis of closely related genomes is best performed using single nucleotide polymorphisms (SNPs) identified by read mapping rather than assembly-based approaches $[6,54,55]$. Many software programs are available for this task; see [56,57] for a review and the updated software list hosted by the SeqAnswers web forum [58]. The process can be somewhat automated using command-driven pipelines such as Nesoni [59] or graphical-interfaces within the MiSeq or Ion Torrent analysis suites or the web-based Galaxy [60].

Detailed gene content comparisons are generally bestperformed using databases tailored to the bacterial species of interest. An excellent place to start is to explore the web-based tools PATRIC [61] and PGAT [62], which are suitable for biologists with little or no programming skills.

\section{Delving deeper into bioinformatics}

For biologists interested in learning more about bioinformatics analysis, we recommend two things. First, get comfortable with the Unix command-line $[63,64]$, which 
opens up a huge array of software tools to do more sophisticated analyses (see [58] for a list of available nextgeneration sequence analysis tools). Second, learn to use the Python scripting language (tutorial at [65]) and associated BioPython functions [66], which will help you to write your own snippets of code to do exactly the analysis you want.

\section{Conclusions}

The bench-top sequencing revolution has led to a 'democratization' of sequencing, meaning most research laboratories can afford to sequence whole bacterial genomes when their work demands it. However analysing the data is now a major bottleneck for most laboratories. We have provided a starting point for biologists to quickly begin working with their own bacterial genome data, without investing money in expensive software or training courses. The figures show examples of what can be achieved with the tools presented, and the accompanying tutorial gives step-by-step instructions for each kind of analysis.

\section{Additional file}

Additional file 1: Tutorial. Bacterial Comparative Genomics Tutorial. Detailed tutorial including worked examples, divided into three sections (1) Genome assembly and annotation, (2) Comparative genome analysis, and (3) Typing and specialist tools.

\section{Competing interests}

The authors declare that they have no competing interests.

\section{Authors' contributions}

DJE and KEH drafted, read and approved the manuscript.

\section{Acknowledgements}

KEH is supported by Fellowship \#628930 from the NHMRC of Australia. DJE is supported by a VLSCI MSc Bioinformatics Studentship from the Victorian Life Sciences Computation Initiative (VLSCI).

\section{Author details}

'Department of Biochemistry and Molecular Biology, Bio21 Institute, University of Melbourne, Victoria 3010, Australia. ${ }^{2}$ Victorian Life Sciences Computation Initiative, University of Melbourne, Victoria 3010, Australia.

\section{Received: 12 February 2013 Accepted: 31 March 2013}

Published: 10 April 2013

\section{References}

1. Loman NJ, Constantinidou C, Chan JZ, Halachev M, Sergeant M, Penn CW, Robinson ER, Pallen MJ: High-throughput bacterial genome sequencing: an embarrassment of choice, a world of opportunity. Nat Rev Microbiol 2012, 10:599-606

2. Stahl PL, Lundeberg J: Toward the single-hour high-quality genome. Annu Rev Biochem 2012, 81:359-378.

3. Howden BP, McEvoy CR, Allen DL, Chua K, Gao W, Harrison PF, Bell J, Coombs G, Bennett-Wood V, Porter JL, et al: Evolution of multidrug resistance during Staphylococcus aureus infection involves mutation of the essential two component regulator WalKR. PLoS Pathog 2011, 7:e1002359.

4. Snitkin ES, Zelazny AM, Thomas PJ, Stock F, Henderson DK, Palmore TN, Segre JA: Tracking a hospital outbreak of carbapenem-resistant Klebsiella pneumoniae with whole-genome sequencing. Sci Transl Med 2012, 4:148ra116.

5. Harris SR, Cartwright EJ, Torok ME, Holden MT, Brown NM, Ogilvy-Stuart AL, Ellington MJ, Quail MA, Bentley SD, Parkhill J, Peacock SJ: Whole-genome sequencing for analysis of an outbreak of meticillin-resistant Staphylococcus aureus: a descriptive study. Lancet Infect Dis 2012, 13:130-136.

6. Holt K, Baker S, Weill F, Holmes E, Kitchen A, Yu J, Sangal V, Brown D, Coia J, Kim D, et al: Shigella sonnei genome sequencing and phylogenetic analysis indicate recent global dissemination from Europe. Nat Genet 2012, 44:1056-1059.

7. Nagarajan N, Cook C, Di Bonaventura M, Ge H, Richards A, Bishop-Lilly KA, DeSalle R, Read TD, Pop M: Finishing genomes with limited resources: lessons from an ensemble of microbial genomes. BMC Genomics 2010, 11:242.

8. Koser CU, Ellington MJ, Cartwright EJ, Gillespie SH, Brown NM, Farrington M, Holden MT, Dougan G, Bentley SD, Parkhill J, Peacock SJ: Routine use of microbial whole genome sequencing in diagnostic and public health microbiology. PLOS Pathog 2012, 8:e1002824.

9. Buchholz U, Bernard H, Werber D, Bohmer MM, Remschmidt C, Wilking H, Delere Y, an der Heiden M, Adlhoch C, Dreesman J, et al: German outbreak of Escherichia coli 0104:H4 associated with sprouts. N Engl J Med 2011, 365:1763-1770.

10. Frank C, Werber D, Cramer JP, Askar M, Faber M, an der Heiden M, Bernard H, Fruth A, Prager R, Spode A, et al: Epidemic profile of Shiga-toxin-producing Escherichia coli O104:H4 outbreak in Germany. N Engl J Med 2011 , 365:1771-1780.

11. Bielaszewska M, Mellmann A, Zhang W, Kock R, Fruth A, Bauwens A, Peters $\mathrm{G}$, Karch $\mathrm{H}$ : Characterisation of the Escherichia coli strain associated with an outbreak of haemolytic uraemic syndrome in Germany, 2011: a microbiological study. Lancet Infect Dis 2011, 11:671-676.

12. Rohde H, Qin J, Cui Y, Li D, Loman NJ, Hentschke M, Chen W, Pu F, Peng Y, Li J, et al: Open-source genomic analysis of Shiga-toxin-producing E. coli 0104:H4. N Engl J Med 2011, 365:718-724.

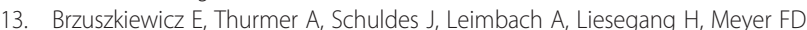
Boelter J, Petersen H, Gottschalk G, Daniel R: Genome sequence analyses of two isolates from the recent Escherichia coli outbreak in Germany reveal the emergence of a new pathotype: Entero-Aggregative -Haemorrhagic Escherichia coli (EAHEC). Arch Microbiol 2011, 193:883-891.

14. Rasko DA, Webster DR, Sahl JW, Bashir A, Boisen N, Scheutz F, Paxinos EE, Sebra $\mathrm{R}$, Chin CS, lliopoulos $\mathrm{D}$, et al: Origins of the $E$. coli strain causing an outbreak of hemolytic-uremic syndrome in Germany. N Engl J Med 2011, 365:709-717.

15. Struelens MJ, Palm D, Takkinen J: Enteroaggregative, Shiga toxinproducing Escherichia coli O104:H4 outbreak: new microbiological findings boost coordinated investigations by European public health laboratories. Euro Surveill 2011, 16:19890.

16. Grad $\mathrm{YH}$, Lipsitch M, Feldgarden M, Arachchi HM, Cerqueira GC, Fitzgerald M, Godfrey P, Haas BJ, Murphy Cl, Russ C, et al: Genomic epidemiology of the Escherichia coli O104:H4 outbreaks in Europe, 2011. Proc Natl Acad Sci U S A 2012, 109:3065-3070.

17. European Nucleotide Archive. [http://www.ebi.ac.uk/ena/data/search? query=0104:h4]

18. Compeau PE, Pevzner PA, Tesler G: How to apply de Bruijn graphs to genome assembly. Nat Biotechnol 2011, 29:987-991.

19. Zerbino DR, Birney E: Velvet: algorithms for de novo short read assembly using de Bruijn graphs. Genome Res 2008, 18:821-829.

20. Zerbino DR, McEwen GK, Margulies EH, Birney E: Pebble and rock band: heuristic resolution of repeats and scaffolding in the velvet short-read de novo assembler. PLoS One 2009, 4:e8407.

21. The MIRA Assembler. [http://sourceforge.net/projects/mira-assembler/]

22. 454 Analysis Software. [http://454.com/products/analysis-software/index.asp]

23. Zerbino DR: Using the Velvet de novo assembler for short-read sequencing technologies. In Current Protocols in Bioinformatics. 11th edition. Edited by Baxevanis AD. US: John Wiley and Sons Inc; 2010. Unit 1115.

24. Velvet Optimiser. [http://bioinformatics.net.au/software.velvetoptimiser.shtml]

25. Kurtz S, Phillippy A, Delcher AL, Smoot M, Shumway M, Antonescu C, Salzberg SL: Versatile and open software for comparing large genomes. Genome Biol 2004, 5:R12.

26. Assefa S, Keane TM, Otto TD, Newbold C, Berriman M: ABACAS: algorithmbased automatic contiguation of assembled sequences. Bioinformatics 2009, 25:1968-1969 
27. Darling AE, Mau B, Perna NT: progressiveMauve: multiple genome alignment with gene gain, loss and rearrangement. PLOS One 2010, 5:e11147.

28. Rissman Al, Mau B, Biehl BS, Darling AE, Glasner JD, Perna NT: Reordering contigs of draft genomes using the Mauve aligner. Bioinformatics 2009, 25:2071-2073.

29. Earl D, Bradnam K, St John J, Darling A, Lin D, Fass J, Yu HO, Buffalo V, Zerbino DR, Diekhans $M$, et al: Assemblathon 1: a competitive assessment of de novo short read assembly methods. Genome Res 2011, 21:2224-2241.

30. Salzberg SL, Phillippy AM, Zimin A, Puiu D, Magoc T, Koren S, Treangen TJ, Schatz MC, Delcher AL, Roberts M, et al: GAGE: A critical evaluation of genome assemblies and assembly algorithms. Genome Res 2012, 22:557-567.

31. Darling AE, Tritt A, Eisen JA, Facciotti MT: Mauve assembly metrics. Bioinformatics 2011, 27:2756-2757.

32. Carver TJ, Rutherford KM, Berriman M, Rajandream MA, Barrell BG, Parkhill J: ACT: the Artemis Comparison Tool. Bioinformatics 2005, 21:3422-3423.

33. Rice $P$, Longden I, Bleasby A: EMBOSS: the European Molecular Biology Open Software Suite. Trends Genet 2000, 16:276-277.

34. RAST (Rapid Annotation using Subsystem Technology). [http://rast.nmpdr.org/]

35. Aziz RK, Bartels D, Best AA, DeJongh M, Disz T, Edwards RA, Formsma K, Gerdes S, Glass EM, Kubal M, et al: The RAST Server: rapid annotations using subsystems technology. BMC Genomics 2008, 9:75.

36. Prokka. [http://www.vicbioinformatics.com/software.prokka.shtml]

37. Stewart AC, Osborne B, Read TD: DIYA: a bacterial annotation pipeline for any genomics lab. Bioinformatics 2009, 25:962-963.

38. Otto TD, Dillon GP, Degrave WS, Berriman M: RATT: Rapid Annotation Transfer Tool. Nucleic Acids Res 2011, 39:e57.

39. Pareja-Tobes P, Manrique M, Pareja-Tobes E, Pareja E, Tobes R: BG7: a new approach for bacterial genome annotation designed for next generation sequencing data. PLoS One 2012, 7:e49239.

40. ACT: Artemis Comparison Tool. [http://www.sanger.ac.uk/resources/software/ act/]

41. Mauve Genome Alignment Software. [http://asap.ahabs.wisc.edu/mauve/]

42. BLAST Ring Image Generator (BR/G). [http://brig.sourceforge.net/]

43. Alikhan NF, Petty NK, Ben Zakour NL, Beatson SA: BLAST Ring Image Generator (BRIG): simple prokaryote genome comparisons. $B M C$ Genomics 2011, 12:402.

44. Zankari E, Hasman H, Cosentino S, Vestergaard M, Rasmussen S, Lund O, Aarestrup FM, Larsen MV: Identification of acquired antimicrobial resistance genes. J Antimicrob Chemother 2012, 67:2640-2644.

45. ResFinder 1.3 (Acquired antimicrobial resistance gene finder). [http://cge.cbs. dtu.dk/services/ResFinder/]

46. Maiden MC: Multilocus sequence typing of bacteria. Annu Rev Microbiol 2006, 60:561-588.

47. Plasmid MLST Databases. [http://pubmlst.org/plasmid/]

48. MLST 1.5 (Multilocus Sequence Typing). [http://cge.cbs.dtu.dk/services/MLST/]

49. SRST on Sourceforge. [http://srst.sourceforge.net]

50. Inouye M, Conway TC, Zobel J, Holt KE: Short read sequence typing (SRST): multi-locus sequence types from short reads. BMC Genomics 2012, 13:338.

51. PHAST (PHAge Search Tool). [http://phast.wishartlab.com/].

52. PATRIC Blast Search. [http://www.patricbrc.org/portal/portal/patric/Blast]

53. NCBI BLAST Server. [http://blast.ncbi.nlm.nih.gov]

54. Croucher NJ, Harris SR, Fraser C, Quail MA, Burton J, van der Linden M, McGee L, von Gottberg A, Song JH, Ko KS, et al: Rapid pneumococcal evolution in response to clinical interventions. Science 2011, 331:430-434.

55. Harris SR, Feil EJ, Holden MT, Quail MA, Nickerson EK, Chantratita N, Gardete S, Tavares A, Day N, Lindsay JA, et al: Evolution of MRSA during hospital transmission and intercontinental spread. Science 2010, 327:469-474.

56. Li H, Homer N: A survey of sequence alignment algorithms for nextgeneration sequencing. Brief Bioinform 2010, 11:473-483.

57. Pabinger $S$, Dander A, Fischer $M$, Snajder R, Sperk $M$, Efremova M, Krabichler B, Speicher MR, Zschocke J, Trajanoski Z: A survey of tools for variant analysis of next-generation genome sequencing data. Brief Bioinform 2013, 14:56-66.

58. SEQanswers Wiki Software. [http://seqanswers.com/wiki/Software]

59. Nesoni. [http://www.vicbioinformatics.com/software.nesoni.shtml]

60. Galaxy - Data intensive biology for everyone. [http://galaxyproject.org/]

61. PATRIC - Pathogen Resource Integration Center. [http://www.patricbrc.org/]

62. PGAT - Prokaryotic Genome Analysis Tool. [http://tools.nwrce.org/pgat/]
63. Software Carpentry - The Shell. [http://software-carpentry.org/4_0/shell/]

64. Stein LD: Unix survival guide. In Current Protocols in Bioinformatics. Edited by Baxevanis AD, et al. US: John Wiley and Sons Inc; 2007. Appendix 1: Appendix 1C.

65. Bassi S: A primer on python for life science researchers. PLoS Comput Biol 2007, 3:e199.

66. Cock PJ, Antao T, Chang JT, Chapman BA, Cox CJ, Dalke A, Friedberg I, Hamelryck T, Kauff F, Wilczynski B, de Hoon MJ: Biopython: freely available Python tools for computational molecular biology and bioinformatics. Bioinformatics 2009, 25:1422-1423.

doi:10.1186/2042-5783-3-2

Cite this article as: Edwards and Holt: Beginner's guide to comparative bacterial genome analysis using next-generation sequence data. Microbial Informatics and Experimentation 2013 3:2

\section{Submit your next manuscript to BioMed Central and take full advantage of:}

- Convenient online submission

- Thorough peer review

- No space constraints or color figure charges

- Immediate publication on acceptance

- Inclusion in PubMed, CAS, Scopus and Google Scholar

- Research which is freely available for redistribution

Submit your manuscript at www.biomedcentral.com/submit
C) Biomed Central 